\title{
On limit distribution of the Matsumoto zeta-function
}

by

\author{
A. LAURINČIKAS (Vilnius) \\ In honour of Professor J. W. S. Cassels \\ on his 75th birthday
}

In [5] K. Matsumoto considered a certain zeta-function $\varphi(s)$ and proved limit theorems in the complex plane $\mathbb{C}$ for it. Let

$$
A_{m}(x)=\prod_{j=1}^{g(m)}\left(1-a_{m}^{(j)} x^{f(j, m)}\right) .
$$

Here $g(m)$ is a positive integer, $a_{m}^{(j)}$ are complex numbers and $f(j, m)$ natural numbers, $1 \leq j \leq g(m), m \in \mathbb{N}$, where $\mathbb{N}$ stands for the set of all natural numbers. Moreover, let $s=\sigma+i t$ be a complex variable, and let $p_{m}$ denote the $m$ th prime number. Define

$$
\varphi(s)=\prod_{m=1}^{\infty} A_{m}^{-1}\left(p_{m}^{-s}\right)
$$

In [5] it is assumed that

$$
g(m) \leq c p_{m}^{\alpha}, \quad\left|a_{m}^{(j)}\right| \leq p_{m}^{\beta}
$$

with a positive constant $c$ and non-negative constants $\alpha$ and $\beta$. The paper [5] contains two limit theorems for $\log \varphi\left(\sigma_{0}+i t\right)$. The first of them examines the case $\sigma_{0}>\alpha+\beta+1$, and the second, under some additional conditions on $\varphi(s)$, concerns the case $\varrho<\sigma_{0} \leq \alpha+\beta+1$, where $\varrho$ is a constant with $\alpha+\beta+1 / 2 \leq \varrho<\alpha+\beta+1$. It is an interesting problem to study the limit distribution of $\varphi(s)$. This was done in [6], where the upper and lower bounds for this limit distribution were obtained.

In [3] we have given a generalization of the results from [5]. We have proved two functional limit theorems with weight for $\varphi(s)$. Let $G$ be a region of the complex plane, and let $\mathbb{C}_{\infty}$ stand for the Riemann sphere 
with spherical metric $d$. Denote by $H(G)$ the space of analytic functions $f: G \rightarrow\left(\mathbb{C}_{\infty}, d\right)$ equipped with the topology of uniform convergence on compacta. Moreover, let $T_{0}$ be a fixed positive number, and let $w(\tau)$ be a positive function of bounded variation on $\left[T_{0}, \infty\right)$. Let $D_{1}=\{s \in \mathbb{C}$ : $\sigma>\alpha+\beta+1\}$. Define

$$
U=U(T, w)=\int_{T_{0}}^{T} w(\tau) d \tau
$$

suppose that $\lim _{T \rightarrow \infty} U(T, w)=\infty$ and define a probability measure

$$
P_{T, w}(A)=\frac{1}{U} \int_{T_{0}}^{T} w(\tau) I_{\{\tau: \varphi(s+i \tau) \in A\}} d \tau, \quad A \in \mathcal{B}\left(H\left(D_{1}\right)\right) .
$$

Here $I_{A}$ denotes the indicator function of the set $A$, and $\mathcal{B}(S)$ stands for the class of Borel sets of the space $S$. In [3] the following assertion has been obtained.

Theorem A. There is a probability measure $P_{w}$ on $\left(H\left(D_{1}\right), \mathcal{B}\left(H\left(D_{1}\right)\right)\right)$ such that the measure $P_{T, w}$ converges weakly to $P_{w}$ as $T \rightarrow \infty$.

Theorem 2 of [3] concerns the behaviour of $\varphi(s)$ in the half-plane $\sigma>$ $\alpha+\beta+1 / 2$.

It is of interest to find the explicit form of the limit measure in Theorem A. B. Bagchi [1] applied ergodic theory to identify limit measures. Unfortunately, we do not know an ergodic theorem with weight $w(\tau)$. Therefore we must introduce some additional condition on the function $w(\tau)$. Denote by $E \xi$ the mean of the random variable $\xi$. Let $X(\tau, \omega)$ be an ergodic process, $\tau \in \mathbb{R}, \omega \in \widetilde{\Omega}$, with $E|X(\tau, \omega)|<\infty$, and with sample paths integrable almost surely in the Riemann sense over every finite interval. Suppose that the function $w(\tau)$ satisfies the relation

$$
\frac{1}{U} \int_{T_{0}}^{T} w(\tau) X(\tau, \omega) d \tau=E X(0, \omega)+o(1)
$$

almost surely as $T \rightarrow \infty$. The latter relation is a generalization of the classical Birkhoff-Khinchin theorem which asserts that

$$
\lim _{T \rightarrow \infty} \frac{1}{T} \int_{0}^{T} X(\tau, \omega) d \tau=E X(0, \omega)
$$

almost surely.

Denote by $\gamma$ the unit circle on the complex plane, that is, $\gamma=\{s \in \mathbb{C}$ : $|s|=1\}$. Moreover, let

$$
\Omega=\prod_{p} \gamma_{p}
$$


where $\gamma_{p}=\gamma$ for all primes $p$. With the product topology and pointwise multiplication the infinite-dimensional torus $\Omega$ is a compact topological group. Therefore there exists a probability Haar measure $m$ on $(\Omega, \mathcal{B}(\Omega))$. Thus we obtain the probability space $(\Omega, \mathcal{B}(\Omega), m)$. Let $\omega(p)$ stand for the projection of $\omega \in \Omega$ to the coordinate space $\gamma_{p}$. Then, setting

$$
\omega(k)=\prod_{p^{\alpha} \| k} \omega^{\alpha}(p),
$$

where $p^{\alpha} \| k$ means that $p^{\alpha} \mid k$ but $p^{\alpha+1} \nmid k$, we obtain an extension of the function $\omega(p)$ to the set of all natural numbers as a completely multiplicative unimodular function.

As noted in [5], $\varphi(s)$ is a holomorphic function in the half-plane $\sigma>$ $\alpha+\beta+1$ with no zeros, and it is represented there by an absolutely convergent Dirichlet series

$$
\varphi(s)=\sum_{k=1}^{\infty} \frac{b(k)}{k^{s}},
$$

where $b(k)=B k^{\alpha+\beta}$. Let $D=\{s \in \mathbb{C}: \sigma>\alpha+\beta+1 / 2\}$, and for $s \in D$ and $\omega \in \Omega$, set

$$
\varphi(s, \omega)=\sum_{k=1}^{\infty} \frac{b(k) \omega(k)}{k^{s}} .
$$

Since, as $N \rightarrow \infty$,

$$
\sum_{k \leq N}|b(k)|^{2}=B N^{2(\alpha+\beta+1 / 2)},
$$

by Lemma 3.4.3 of [1] the series $\varphi(s, \omega)$ converges uniformly on compact subsets of $D$, and $\varphi(s, \omega)$ is an $H(D)$-valued random element defined on the probability space $(\Omega, \mathcal{B}(\Omega), m)$. Let $P_{\varphi}$ denote the distribution of $\varphi(s, \omega)$, and let $P_{1, \varphi}$ be the restriction of $P_{\varphi}$ to $\left(H\left(D_{1}\right), \mathcal{B}\left(H\left(D_{1}\right)\right)\right)$. The aim of this paper is to prove the following result.

TheOREM. Under the assumption (1) the measure $P_{T, w}$ converges weakly to $P_{1, \varphi}$ as $T \rightarrow \infty$.

This theorem shows that the limit measure is independent of the weight function $w(\tau)$. For its proof we will apply the method of [1].

First we state a lemma for trigonometric polynomials

$$
p_{n}(s)=\sum_{k=1}^{n} \frac{a(k)}{k^{s}}, \quad p_{n}(s, g)=\sum_{k=1}^{n} \frac{a(k) g(k)}{k^{s}},
$$

where $g(k)$ is a unimodular completely multiplicative function. Let $G$ be a 
region in $\mathbb{C}$, and

$$
\begin{aligned}
& P_{T, p_{n}, w}(A)=\frac{1}{U} \int_{T_{0}}^{T} w(\tau) I_{\left\{\tau: p_{n}(s+i \tau) \in A\right\}} d \tau, \quad A \in \mathcal{B}(H(G)), \\
& \widetilde{P}_{T, p_{n}, w}(A)=\frac{1}{U} \int_{T_{0}}^{T} w(\tau) I_{\left\{\tau: p_{n}(s+i \tau, g) \in A\right\}} d \tau, \quad A \in \mathcal{B}(H(G)) .
\end{aligned}
$$

Lemma 1. The probability measures $P_{T, p_{n}, w}$ and $\widetilde{P}_{T, p_{n}, w}$ both converge weakly to the same measure as $T \rightarrow \infty$.

Proof. This is Lemma 2 of [4].

Now we will prove a similar assertion to Lemma 1 for the function $\varphi(s)$ in $D_{1}$. For convenience of the reader we recall some probabilistic results.

Let $S$ be a separable metric space with a metric $\varrho$, and let $Y_{n}, X_{1 n}$, $X_{2 n}, \ldots$ be $S$-valued random elements defined on $\left(\Omega_{1}, \mathcal{F}, \mathbb{P}\right)$. The following assertion is Theorem 4.2 of [2].

Lemma 2. Suppose that $X_{k n} \underset{n \rightarrow \infty}{\stackrel{\mathcal{D}}{\longrightarrow}} X_{k}$ for each $k$ and also $X_{k} \underset{k \rightarrow \infty}{\stackrel{\mathcal{D}}{\longrightarrow}} X$. If for every $\varepsilon>0$,

$$
\lim _{k \rightarrow \infty} \limsup _{n \rightarrow \infty} \mathbb{P}\left(\varrho\left(X_{k n}, Y_{n}\right) \geq \varepsilon\right)=0,
$$

then $Y_{n} \underset{n \rightarrow \infty}{\stackrel{\mathcal{D}}{\longrightarrow}} X$.

Now let $P_{n}$ and $P$ be probability measures on $(S, \mathcal{B}(S))$.

Lemma 3. $P_{n}$ converges weakly to $P$ as $n \rightarrow \infty$ if and only if any subsequence $\left\{P_{n^{\prime}}\right\}$ contains another subsequence $\left\{P_{n^{\prime \prime}}\right\}$ such that $P_{n^{\prime \prime}} \rightarrow P$ as $n^{\prime \prime} \rightarrow \infty$.

Proof. This is Theorem 2.3 of [2].

Let $S$ and $S_{1}$ be two metric spaces, and let $h: S \rightarrow S_{1}$ be a measurable function. Then every probability measure $P$ on $(S, \mathcal{B}(S))$ induces a unique probability measure $P h^{-1}$ on $\left(S_{1}, \mathcal{B}\left(S_{1}\right)\right)$ defined by $P h^{-1}(A)=P\left(h^{-1} A\right)$, $A \in \mathcal{B}\left(S_{1}\right)$.

Lemma 4. Let $h: S \rightarrow S_{1}$ be a continuous function, and let $P_{n}$ and $P$ be probability measures on $(S, \mathcal{B}(S))$. Suppose that $P_{n}$ converges weakly to $P$ as $n \rightarrow \infty$. Then $P_{n} h^{-1}$ converges weakly to $P h^{-1}$ as $n \rightarrow \infty$.

Proof. This is a particular case of Theorem 5.1 of [2].

For $\omega \in \Omega$, let

$$
\widetilde{P}_{T, w}(A)=\frac{1}{U} \int_{T_{0}}^{T} w(\tau) I_{\{\tau: \varphi(s+i \tau, \omega) \in A\}} d \tau, \quad A \in \mathcal{B}\left(H\left(D_{1}\right)\right) .
$$


Lemma 5. There exists a probability measure $P_{w}$ on $\left(H\left(D_{1}\right), \mathcal{B}\left(H\left(D_{1}\right)\right)\right)$ such that the measures $P_{T, w}$ and $\widetilde{P}_{T, w}$ both converge weakly to $P_{w}$ as $T \rightarrow \infty$.

Pro of. Let

$$
\varphi_{n}(s)=\sum_{k=1}^{n} \frac{b(k)}{k^{s}}
$$

and, for $\omega \in \Omega$,

$$
\varphi_{n}(s, \omega)=\sum_{k=1}^{n} \frac{b(k) \omega(k)}{k^{s}} .
$$

Define two probability measures

$$
\begin{aligned}
& P_{T, \varphi_{n}, w}(A)=\frac{1}{U} \int_{T_{0}}^{T} w(\tau) I_{\left\{\tau: \varphi_{n}(s+i \tau) \in A\right\}} d \tau, \quad A \in \mathcal{B}\left(H\left(D_{1}\right)\right), \\
& \widetilde{P}_{T, \varphi_{n}, w}(A)=\frac{1}{U} \int_{T_{0}}^{T} w(\tau) I_{\left\{\tau: \varphi_{n}(s+i \tau, \omega) \in A\right\}} d \tau, \quad A \in \mathcal{B}\left(H\left(D_{1}\right)\right) .
\end{aligned}
$$

Then by Lemma 1 both $P_{T, \varphi_{n}, w}$ and $\widetilde{P}_{T, \varphi_{n}, w}$ converge weakly to the same measure $P_{\varphi_{n}, w}$, say, as $T \rightarrow \infty$. We will prove that the family $\left\{P_{\varphi_{n}, w}: n \in \mathbb{N}\right\}$ of probability measures is tight. Let $\eta$ be a random variable on $\left(\Omega_{1}, \mathcal{F}, \mathbb{P}\right)$ with distribution

$$
\mathbb{P}(\eta \in A)=\frac{1}{U} \int_{T_{0}}^{T} w(\tau) I_{A} d \tau, \quad A \in \mathcal{B}(\mathbb{R}) .
$$

We set $X_{T, \varphi_{n}}(s)=\varphi_{n}(s+i \eta)$. Then

$$
X_{T, \varphi_{n}} \underset{T \rightarrow \infty}{\stackrel{\mathcal{D}}{\longrightarrow}} X_{\varphi_{n}}
$$

where $X_{\varphi_{n}}$ is an $H\left(D_{1}\right)$-valued random element with distribution $P_{\varphi_{n}, w}$. Since, for $\sigma>\alpha+\beta+1$, the Dirichlet series for $\varphi(s)$ is absolutely convergent, it follows that

$$
\sup _{n \geq 1} \limsup _{T \rightarrow \infty} \frac{1}{U} \int_{T_{0}}^{T} w(\tau) \sup _{s \in K_{l}}\left|\varphi_{n}(s+i \tau)\right| d \tau \leq R_{l}<\infty,
$$

where $\left\{K_{l}\right\}$ is a sequence of compact subsets of $D_{1}$ such that $D_{1}=\bigcup_{l=1}^{\infty} K_{l}$, $K_{l} \subset K_{l+1}, l=1,2, \ldots$, and if $K$ is a compact set and $K \subset D_{1}$ then $K \subseteq K_{l}$ for some $l$. Let $\varepsilon>0$. Then, setting $M_{l}=R_{l} 2^{l} \varepsilon^{-1}$, we find that

$$
\begin{aligned}
\limsup _{T \rightarrow \infty} \mathbb{P}\left(\sup _{s \in K_{l}}\left|X_{T, \varphi_{n}}(s)\right|>M_{l}\right) \\
\quad \leq \frac{1}{M_{l}} \sup _{n \geq 1} \limsup _{T \rightarrow \infty} \frac{1}{U} \int_{T_{0}}^{T} w(\tau) \sup _{s \in K_{l}}\left|\varphi_{n}(s+i \tau)\right| d \tau \leq \varepsilon / 2^{l}
\end{aligned}
$$


for all $l \in \mathbb{N}$. Define $h: H\left(D_{1}\right) \rightarrow \mathbb{R}$ by

$$
h(f)=\sup _{s \in K_{l}}|f(s)|, \quad f \in H\left(D_{1}\right) .
$$

Then $h$ is continuous, and thus by (2) and Lemma 4 ,

$$
\sup _{s \in K_{l}}\left|X_{T, \varphi_{n}}(s)\right| \underset{T \rightarrow \infty}{\stackrel{\mathcal{D}}{\longrightarrow}} \sup _{s \in K_{l}}\left|X_{\varphi_{n}}(s)\right| .
$$

This together with (3) yields

$$
\mathbb{P}\left(\sup _{s \in K_{l}}\left|X_{\varphi_{n}}(s)\right|>M_{l}\right) \leq \varepsilon / 2^{l}
$$

for all $l \in \mathbb{N}$. Define

$$
H_{\varepsilon}=\left\{f \in H\left(D_{1}\right): \sup _{s \in K_{l}}|f(s)| \leq M_{l}, l \geq 1\right\} .
$$

Then $H_{\varepsilon}$ is a set of functions analytic on $D_{1}$ and uniformly bounded on every compact $K \subset D_{1}$, and therefore, by the compactness principle, it is a compact subset of $H\left(D_{1}\right)$. The inequality (4) implies $\mathbb{P}\left(X_{\varphi_{n}}(s) \in H_{\varepsilon}\right) \geq 1-\varepsilon$ for all $n \geq 1$, or, since $P_{\varphi_{n}, w}$ is the distribution of $X_{\varphi_{n}}, P_{\varphi_{n}, w}\left(H_{\varepsilon}\right) \geq 1-\varepsilon$ for all $n \geq 1$. So we have proved that the family $\left\{P_{\varphi_{n}, w}\right\}$ is tight. Hence by the Prokhorov theorem it is relatively compact.

Let

$$
\varrho_{1}\left(f_{1}, f_{2}\right)=\sum_{l=1}^{\infty} 2^{-l} \frac{\varrho_{1, l}\left(f_{1}, f_{2}\right)}{1+\varrho_{1, l}\left(f_{1}, f_{2}\right)}
$$

where

$$
\varrho_{1, l}\left(f_{1}, f_{2}\right)=\sup _{s \in K_{l}}\left|f_{1}(s)-f_{2}(s)\right|, \quad f_{1}, f_{2} \in H\left(D_{1}\right) .
$$

Then $\varrho_{1}\left(f_{1}, f_{2}\right)$ is a metric on $H\left(D_{1}\right)$. Since $\varphi_{n}(s) \rightarrow \varphi(s)$ as $n \rightarrow \infty$ uniformly on compact subsets of $D_{1}$, we have, for every $\varepsilon>0$,

(5) $\quad \lim _{n \rightarrow \infty} \limsup _{T \rightarrow \infty} \frac{1}{U} \int_{T_{0}}^{T} w(\tau) I_{\left\{\tau: \varrho_{1}\left(\varphi(s+i \tau), \varphi_{n}(s+i \tau)\right) \geq \varepsilon\right\}} d \tau$

$$
\leq \lim _{n \rightarrow \infty} \limsup _{T \rightarrow \infty} \frac{1}{\varepsilon U} \int_{T_{0}}^{T} w(\tau) \varrho_{1}\left(\varphi(s+i \tau), \varphi_{n}(s+i \tau)\right) d \tau=0 .
$$

Now set

$$
X_{T}(s)=\varphi(s+i \eta) .
$$

Then (5) can be written as

$$
\lim _{n \rightarrow \infty} \limsup _{T \rightarrow \infty} \mathbb{P}\left(\varrho_{1}\left(X_{T, \varphi_{n}}(s), X_{T}(s)\right) \geq \varepsilon\right)=0 .
$$


Since the family $\left\{P_{\varphi_{n}, w}\right\}$ is relatively compact, there exists a subsequence $\left\{P_{\varphi_{n^{\prime}}, w}\right\}$ which converges weakly to $P_{w}$, say, as $n \rightarrow \infty$. Then, obviously,

$$
X_{\varphi_{n^{\prime}}} \underset{n^{\prime} \rightarrow \infty}{\stackrel{\mathcal{D}}{\longrightarrow}} P_{w} .
$$

Hence and from (6) and (2), using Lemma 2, we obtain

$$
X_{T} \underset{T \rightarrow \infty}{\stackrel{\mathcal{D}}{\longrightarrow}} P_{w} .
$$

This means that there is a probability measure $P_{w}$ on $\left(H\left(D_{1}\right), \mathcal{B}\left(H\left(D_{1}\right)\right)\right)$ such that $P_{T, w}$ converges weakly to $P_{w}$ as $T \rightarrow \infty$. Moreover, (7) shows that $P_{w}$ is independent of the choice of the subsequence $\left\{P_{n^{\prime}, w}\right\}$. Thus by Lemma 3,

$$
X_{\varphi_{n}} \underset{n \rightarrow \infty}{\stackrel{\mathcal{D}}{\longrightarrow}} P_{w} .
$$

Now, for $\omega \in \Omega$, let

$$
\widetilde{X}_{T, \varphi_{n}}(s, \omega)=\varphi_{n}(s+i \eta, \omega), \quad \widetilde{X}_{T}(s, \omega)=\varphi(s+i \eta, \omega) .
$$

Then, reasoning as above and using (8), we conclude that the measure $\widetilde{P}_{T, w}$ also converges weakly to $P_{w}$ as $T \rightarrow \infty$.

We precede the proof of the Theorem by some remarks on ergodic theory.

Let $\mathcal{P}$ denote the set of all prime numbers, and let $a_{\tau}=\left\{p^{-i \tau}: p \in \mathcal{P}\right\}$ for $\tau \in \mathbb{R}$. Then $\left\{a_{\tau}: \tau \in \mathbb{R}\right\}$ is a one-parameter group. Define a oneparameter family $\left\{g_{\tau}: \tau \in \mathbb{R}\right\}$ of measurable transformations of $\Omega$ by $g_{\tau}(\omega)=a_{\tau} \omega$ for $\omega \in \Omega$. A set $A \in \mathcal{B}(\Omega)$ is called invariant with respect to the group $\left\{g_{\tau}: \tau \in \mathbb{R}\right\}$ if for each $\tau$ the sets $A$ and $A_{\tau}=g_{\tau}(A)$ differ by a set of zero $m$-measure. In other words, $m\left(A \Delta A_{\tau}\right)=0$, where $\triangle$ denotes a symmetric difference. All invariant sets form a $\sigma$-field. A one-parameter group $\left\{g_{\tau}: \tau \in \mathbb{R}\right\}$ is called ergodic if its $\sigma$-field of invariant sets consists only of sets having $m$-measure equal to 0 or 1 .

Lemma 6. The one-parameter group $\left\{g_{\tau}: \tau \in \mathbb{R}\right\}$ is ergodic.

Proof. This is Lemma 3.4.2 of [1].

Proof of Theorem. By Lemma 5 the measures $P_{T, w}$ and $\widetilde{P}_{T, w}$ converge weakly to the same measure $P_{w}$ as $T \rightarrow \infty$. It remains to prove that $P_{w}=P_{1, \varphi}$.

Let $A \in \mathcal{B}\left(H\left(D_{1}\right)\right)$ be a continuity set of $P_{w}$. Then, by Lemma 5 ,

$$
\lim _{T \rightarrow \infty} \frac{1}{U} \int_{T_{0}}^{T} w(\tau) I_{\{\tau: \varphi(s+i \tau, \omega) \in A\}} d \tau=P_{w}(A) .
$$


Fix $A$ and define a random variable $\theta$ on $(\Omega, \mathcal{B}(\Omega))$ by

$$
\theta(\omega)= \begin{cases}1 & \text { if } \varphi(s, \omega) \in A \\ 0 & \text { if } \varphi(s, \omega) \notin A .\end{cases}
$$

Clearly,

$$
E(\theta)=\int_{\Omega} \theta d m=m\{\omega: \varphi(s, \omega) \in A\}=P_{1, \varphi}(A)<\infty .
$$

It follows from Lemma 6 that $\theta\left(g_{\tau}(\omega)\right)$ is an ergodic process. Therefore, by (1)

$$
\lim _{T \rightarrow \infty} \frac{1}{U} \int_{T_{0}}^{T} w(\tau) \theta\left(g_{\tau}(\omega)\right) d \tau=E(\theta)
$$

for almost all $\omega \in \Omega$. On the other hand,

$$
\begin{aligned}
\frac{1}{U} \int_{T_{0}}^{T} w(\tau) \theta\left(g_{\tau}(\omega)\right) d \tau & =\frac{1}{U} \int_{T_{0}}^{T} w(\tau) I_{\left\{\tau: \varphi\left(s, g_{\tau}(\omega)\right) \in A\right\}} d \tau \\
& =\frac{1}{U} \int_{T_{0}}^{T} w(\tau) I_{\{\tau: \varphi(s+i \tau, \omega) \in A\}} d \tau .
\end{aligned}
$$

From this, (10), and (11) we obtain

$$
\lim _{T \rightarrow \infty} \frac{1}{U} \int_{T_{0}}^{T} w(\tau) I_{\{\tau: \varphi(s+i \tau, \omega) \in A\}} d \tau=P_{1, \varphi}(A)
$$

for almost all $\omega \in \Omega$. Thus in view of $(9), P_{w}(A)=P_{1, \varphi}(A)$ for any continuity set of the measure $P_{w}$. This implies that $P_{w}(A)=P_{1, \varphi}(A)$ for all $A \in \mathcal{B}\left(H\left(D_{1}\right)\right)$, since the continuity sets constitute a determining class. The Theorem is proved.

Now let $P$ denote the limit measure in the theorem of Matsumoto [5], i.e., for $\sigma_{0}>\alpha+\beta+1, \lim _{T \rightarrow \infty}(1 / T)$ meas $\left\{t \in[0, T]: \log \varphi\left(\sigma_{0}+i t\right) \in\right.$ $A\}=P(A)$ for all continuity sets $A$ of $P$. Our Theorem allows us to identify the measure $P$.

Corollary. We have, for $\sigma_{0}>\alpha+\beta+1$,

$$
P(A)=m\left(\log \varphi\left(\sigma_{0}, \omega\right) \in A\right), \quad A \in \mathcal{B}(\mathbb{C}) .
$$

Proof. Let $h: H\left(D_{1}\right) \rightarrow \mathbb{C}$ be given by $h(f)=\log f\left(\sigma_{0}\right)$ for $f \in H\left(D_{1}\right)$. Then the weak convergence of the measure $P_{T, w}$ to $P_{1, \varphi}$ (in this case $w(\tau) \equiv 1)$ together with Lemma 4 implies the weak convergence of $P_{T, 1} h^{-1}$ to $P_{1, \varphi} h^{-1}$. This proves the Corollary. 
The research described in this publication was made possible in part by Grant No LI 2100 from the Joint Fund Program of Lithuanian Government and International Science Foundation.

\section{References}

[1] B. Bagchi, The statistical behaviour and universality properties of the Riemann zetafunction and other allied Dirichlet series, Ph.D. Thesis, Calcutta, Indian Statistical Institute, 1981.

[2] P. Billingsley, Convergence of Probability Measures, Wiley, New York, 1968.

[3] A. Laurinčikas, Limit theorems for the Matsumoto zeta-function, J. Théor. Nombres Bordeaux, to appear.

[4] A. Laurinčikas and G. Misevičius, On limit distribution of the Riemann zetafunction, Acta Arith. 76 (1996), 317-334.

[5] K. Matsumoto, Value-distribution of zeta functions, in: Lecture Notes in Math. 1434, Springer, 1990, 178-187.

[6] - On the magnitude of asymptotic probability measures of Dedekind zeta-functions and other Euler products, Acta Arith. 60 (1991), 125-147.

Department of Mathematics

Vilnius University

Naugarduko, 24

2006 Vilnius, Lithuania

E-mail: antanas.laurincikas@maf.vu.lt

Received on 12.9.1995

and in revised form on 30.11.1995

$(2860)$ 